\title{
Poly-Cauchy numbers and polynomials of the second kind with umbral calculus viewpoint
}

Dae San Kim ${ }^{1}$ and Taekyun Kim²*

"Correspondence: tkkim@kw.ac.kr ${ }^{2}$ Department of Mathematics, Kwangwoon University, Seoul, 139-701, Republic of Korea Full list of author information is available at the end of the article

\section{Abstract}

In this paper, we consider the poly-Cauchy polynomials and numbers of the second kind which were studied by Komatsu. We note that the poly-Cauchy polynomials of the second kind are the special generalized Bernoulli polynomials of the second kind. The purpose of this paper is to give various identities of the poly-Cauchy polynomials of the second kind which are derived from umbral calculus.

\section{Introduction}

As is well known, the Bernoulli polynomials of the second kind are defined by the generating function to be

$$
\frac{t}{\log (1+t)}(1+t)^{x}=\sum_{n=0}^{\infty} b_{n}(x) \frac{t^{n}}{n !} \quad \text { (see [1, p.130]). }
$$

When $x=0, b_{n}=b_{n}(0)$ are called the Bernoulli numbers of the second kind (see [1, p.131]).

Let $\operatorname{Lif}_{k}(x)$ be the polylogarithm factorial function, which is defined by

$$
\operatorname{Lif}_{k}(x)=\sum_{n=0}^{\infty} \frac{x^{m}}{m !(m+1)^{k}} \quad(\text { see }[2-7])
$$

The poly-Cauchy polynomials of the second kind $\widehat{c}_{n}^{(k)}(x)\left(k \in \mathbf{Z}, n \in \mathbf{Z}_{\geq 0}\right)$ are defined by the generating function to be

$$
\operatorname{Lif}_{k}(-\log (1+t))(1+t)^{x}=\sum_{n=0}^{\infty} \widehat{c}_{n}^{(k)}(x) \frac{t^{n}}{n !} \quad(\text { see }[2,3])
$$

When $x=0, \widehat{c}_{n}^{(k)}=\widehat{c}_{n}^{(k)}(0)$ are called the poly-Cauchy numbers of the second kind, defined by

$$
\sum_{n=0}^{\infty} \widehat{c}_{n}^{(k)} \frac{t^{n}}{n !}=\operatorname{Lif}_{k}(-\log (1+t))
$$

In particular, if we take $k=1$, then we have

$$
\operatorname{Lif}_{1}(-\log (1+t))(1+t)^{x}=\frac{t}{(1+t) \log (1+t)}(1+t)^{x}=\frac{t(1+t)^{x-1}}{\log (1+t)} .
$$

(02014 Kim and Kim; licensee Springer. This is an Open Access article distributed under the terms of the Creative Commons Attribution License (http://creativecommons.org/licenses/by/2.0), which permits unrestricted use, distribution, and reproduction in any medium, provided the original work is properly cited. 
Thus, we note that

$$
\widehat{c}_{n}^{(1)}(x)=b_{n}(x-1)=B_{n}^{(n)}(x),
$$

where $B_{n}^{(\alpha)}(x)$ are the Bernoulli polynomials of order $\alpha$ (see [8]) as their numbers [9, p.257 and p.259].

When $x=0, \widehat{c}_{n}^{(1)}=\widehat{c}_{n}^{(1)}(0)=b_{n}(-1)=B_{n}^{(n)}$, where $B_{n}^{(\alpha)}$ are the Bernoulli numbers of order $\alpha$.

The falling factorial is defined by

$$
(x)_{n}=x(x-1) \cdots(x-n+1)=\sum_{l=0}^{n} S_{1}(n, l) x^{l},
$$

where $S_{1}(n, l)$ is the signed Stirling number of the first kind.

For $m \in \mathbf{Z}_{\geq 0}$, it is well known that

$$
\begin{aligned}
(\log (1+t))^{m} & =m ! \sum_{l=m}^{\infty} S_{1}(l, m) \frac{t^{l}}{l !} \\
& \left.=\sum_{l=0}^{\infty} S_{1}(l+m, m) \frac{m !}{(l+m) !} t^{l+m} \quad \text { (see }[10, \mathrm{p} .62]\right)
\end{aligned}
$$

For $\lambda \in \mathbf{C}$ with $\lambda \neq 1$, the Frobenius-Euler polynomials of order $r$ are defined by the generating function to be

$$
\left(\frac{1-\lambda}{e^{t}-\lambda}\right)^{r} e^{x t}=\sum_{n=0}^{\infty} H_{n}^{(r)}(x \mid \lambda) \frac{t^{n}}{n !} \quad(\text { see }[11-13]) .
$$

In this paper, we investigate the properties of the poly-Cauchy numbers and polynomials of the second kind with umbral calculus viewpoint. The purpose of this paper is to give various identities of the poly-Cauchy polynomials of the second kind which are derived from umbral calculus.

\section{Umbral calculus}

Let $\mathbf{C}$ be the complex number field and let $\mathcal{F}$ be the set of all formal power series in the variable $t$ :

$$
\mathcal{F}=\left\{f(t)=\sum_{k=0}^{\infty} \frac{a_{k}}{k !} t^{k} \mid a_{k} \in \mathbf{C}\right\}
$$

Let $\mathbb{P}=\mathbf{C}[x]$ and let $\mathbb{P}^{*}$ be the vector space of all linear functionals on $\mathbb{P} .\langle L \mid p(x)\rangle$ is the action of the linear functional $L$ on the polynomial $p(x)$, and we recall that the vector space operations on $\mathbb{P}^{*}$ are defined by $\langle L+M \mid p(x)\rangle=\langle L \mid p(x)\rangle+\langle M \mid p(x)\rangle,\langle c L \mid p(x)\rangle=c\langle L \mid p(x)\rangle$, where $c$ is a complex constant in $\mathbf{C}$. For $f(t) \in \mathcal{F}$, let us define the linear functional on $\mathbb{P}$ by setting

$$
\left\langle f(t) \mid x^{n}\right\rangle=a_{n} \quad(n \geq 0) .
$$


Then, by (9) and (10), we get

$$
\left\langle t^{k} \mid x^{n}\right\rangle=n ! \delta_{n, k} \quad(n, k \geq 0)
$$

where $\delta_{n, k}$ is Kronecker's symbol.

For $f_{L}(t)=\sum_{k=0}^{\infty} \frac{\left\langle L \mid x^{k}\right\rangle}{k !} t^{k}$, we have $\left\langle f_{L}(t) \mid x^{n}\right\rangle=\left\langle L \mid x^{n}\right\rangle$. That is, $L=f_{L}(t)$. The map $L \longmapsto f_{L}(t)$ is a vector space isomorphism from $\mathbb{P}^{*}$ onto $\mathcal{F}$. Henceforth, $\mathcal{F}$ denotes both the algebra of formal power series in $t$ and the vector space of all linear functionals on $\mathbb{P}$, and so an element $f(t)$ of $\mathcal{F}$ will be thought of as both a formal power series and a linear functional. We call $\mathcal{F}$ the umbral algebra and the umbral calculus is the study of umbral algebra. The order $O(f(t))$ of a power series $f(t)(\neq 0)$ is the smallest integer $k$ for which the coefficient of $t^{k}$ does not vanish. If $O(f(t))=1$, then $f(t)$ is called a delta series; if $O(f(t))=0$, then $f(t)$ is called an invertible series (see [10, 14, 15]). For $f(t), g(t) \in \mathcal{F}$ with $O(f(t))=1$ and $O(g(t))=0$, there exists a unique sequence $s_{n}(x)\left(\operatorname{deg} s_{n}(x)=n\right)$ such that $\left\langle g(t) f(t)^{k} \mid s_{n}(x)\right\rangle=$ $n ! \delta_{n, k}$ for $n, k \geq 0$. The sequence $s_{n}(x)$ is called the Sheffer sequence for $(g(t), f(t))$ which is denoted by $s_{n}(x) \sim(g(t), f(t))$ (see $[10,15]$ ).

For $f(t), g(t) \in \mathcal{F}$ and $p(x) \in \mathbb{P}$, we have

$$
\langle f(t) g(t) \mid p(x)\rangle=\langle f(t) \mid g(t) p(x)\rangle=\langle g(t) \mid f(t) p(x)\rangle
$$

and

$$
f(t)=\sum_{k=0}^{\infty}\left\langle f(t) \mid x^{k}\right\rangle \frac{t^{k}}{k !}, \quad p(x)=\sum_{k=0}^{\infty}\left\langle t^{k} \mid p(x)\right\rangle \frac{x^{k}}{k !} .
$$

Thus, by (13), we get

$$
t^{k} p(x)=p^{(k)}(x)=\frac{d^{k} p(x)}{d x^{k}}, \quad \text { and } \quad e^{y t} p(x)=p(x+y) .
$$

Let us assume that $s_{n}(x) \sim(g(t), f(t))$. Then the generating function of $s_{n}(x)$ is given by

$$
\frac{1}{g(\bar{f}(t))} e^{\bar{x}(t)}=\sum_{n=0}^{\infty} s_{n}(x) \frac{t^{n}}{n !}, \quad \text { for all } x \in \mathbf{C}
$$

where $\bar{f}(t)$ is the compositional inverse of $f(t)$ with $\bar{f}(f(t))=t$ (see $[10,15]$ ).

For $s_{n}(x) \sim(g(t), f(t))$, we have the following equation:

$$
\begin{aligned}
& f(t) s_{n}(x)=n s_{n-1}(x) \quad(n \geq 0), \\
& s_{n}(x)=\sum_{j=0}^{n} \frac{1}{j !}\left|g(\bar{f}(t))^{-1} \bar{f}(t)^{j}\right| x^{n} \mid x^{j},
\end{aligned}
$$

and

$$
s_{n}(x+y)=\sum_{j=0}^{n}\left(\begin{array}{l}
n \\
j
\end{array}\right) s_{j}(x) p_{n-j}(y)
$$

where $p_{n}(x)=g(t) s_{n}(x)($ see $[10$, p.21] $)$. 
Let us assume that $p_{n}(x) \sim(1, f(t)), q_{n}(x) \sim(1, g(t))$. Then the transfer formula is given by

$$
q_{n}(x)=x\left(\frac{f(t)}{g(t)}\right)^{n} x^{-1} p_{n}(x) \quad(n \geq 0)(\text { see }[10, \mathrm{p} .51])
$$

For $s_{n}(x) \sim(g(t), f(t)), r_{n}(x) \sim(h(t), l(t))$, let us assume that

$$
s_{n}(x)=\sum_{m=0}^{n} C_{n, m} r_{n}(x) \quad(n \geq 0)
$$

Then we have

$$
C_{n, m}=\frac{1}{m !}\left\langle\frac{h(\bar{f}(t))}{g(\bar{f}(t))} l(\bar{f}(t))^{m} \mid x^{n}\right\rangle \quad \text { (see [10, p.132]). }
$$

\section{Poly-Cauchy numbers and polynomials of the second kind}

From (3), we note that $\widehat{c}_{n}^{(k)}(x)$ is the Sheffer sequence for the pair

$$
\left(g(t)=\frac{1}{\operatorname{Lif}_{k}(-t)}, f(t)=e^{t}-1\right)
$$

that is,

$$
\widehat{c}_{n}^{(k)}(x) \sim\left(\frac{1}{\operatorname{Lif}_{k}(-t)}, e^{t}-1\right)
$$

Because for $\bar{f}(t)=\log (1+t)$, using the formula (15), we get

$$
\operatorname{Lif}_{k}(-\log (1+t))(1+t)^{x}=\sum_{n=0}^{\infty} s_{n}(x) \frac{t^{n}}{n !}
$$

which is the generating function of $\widehat{c}_{n}^{(k)}(x)$ in (3).

From (21), we have

$$
\frac{1}{\operatorname{Lif}_{k}(-t)} \widehat{c}_{n}^{(k)}(x) \sim\left(1, e^{t}-1\right)
$$

and

$$
(x)_{n}=\sum_{l=0}^{n} S_{1}(n, l) x^{l} \sim\left(1, e^{t}-1\right) .
$$

By (22) and (23), we get

$$
\begin{aligned}
\widehat{c}_{n}^{(k)}(x) & =\operatorname{Lif}_{k}(-t)(x)_{n}=\sum_{m=0}^{n} S_{1}(n, m) \operatorname{Lif}_{k}(-t) x^{m} \\
& =\sum_{m=0}^{n} S_{1}(n, m) \sum_{a=0}^{m} \frac{(-1)^{a}}{a !(a+1)^{k}} t^{a} x^{m}
\end{aligned}
$$




$$
\begin{aligned}
& =\sum_{m=0}^{n} \sum_{a=0}^{m} S_{1}(n, m) \frac{(-1)^{a}\left(\begin{array}{c}
m \\
a
\end{array}\right)}{(a+1)^{k}} x^{m-a} \\
& =\sum_{m=0}^{n} \sum_{j=0}^{m} S_{1}(n, m) \frac{(-1)^{m-j}\left(\begin{array}{c}
m \\
j
\end{array}\right)}{(m-j+1)^{k}} x^{j} \\
& =\sum_{j=0}^{n}\left\{\sum_{m=j}^{n} S_{1}(n, m) \frac{(-1)^{m-j}\left(\begin{array}{c}
m \\
j
\end{array}\right)}{(m-j+1)^{k}}\right\} x^{j} .
\end{aligned}
$$

By (17) and (21), we get

$$
\widehat{c}_{n}^{(k)}(x)=\sum_{j=0}^{n} \frac{1}{j !}\left\langle\operatorname{Lif}_{k}(-\log (1+t))(\log (1+t))^{j}\left|x^{n}\right| x^{j}\right.
$$

Now, we observe that

$$
\begin{aligned}
\left\langle\operatorname{Lif}_{k}(-\log (1+t))(\log (1+t))^{j} \mid x^{n}\right\rangle \\
=\sum_{m=0}^{\infty} \frac{(-1)^{m}}{m !(m+1)^{k}}\left\langle(\log (1+t))^{m+j} \mid x^{n}\right\rangle \\
\left.=\sum_{m=0}^{n-j} \frac{(-1)^{m}}{m !(m+1)^{k}} \sum_{l=0}^{n-j-m} \frac{S_{1}(l+m+j, m+j)}{(l+m+j) !}(m+j) !\left|t^{m+j+l}\right| x^{n}\right\rangle \\
=\sum_{m=0}^{n-j} \frac{(-1)^{m}}{m !(m+1)^{k}} \sum_{l=0}^{n-m-j} \frac{S_{1}(l+m+j, m+j)}{(l+m+j) !}(m+j) ! n ! \delta_{n, l+m+j} \\
=\sum_{m=0}^{n-j} \frac{(-1)^{m}(m+j) !}{m !(m+1)^{k}} S_{1}(n, m+j) .
\end{aligned}
$$

From (25) and (26), we have

$$
\begin{aligned}
\widehat{c}_{n}^{(k)}(x) & =\sum_{j=0}^{n} \frac{1}{j !} \sum_{m=0}^{n-j} \frac{(-1)^{m}(m+j) !}{m !(m+1)^{k}} S_{1}(n, m+j) x^{j}=\sum_{j=0}^{n}\left\{\sum_{m=0}^{n-j} \frac{(-1)^{m}\left(\begin{array}{c}
m+j \\
m
\end{array}\right)}{(m+1)^{k}} S_{1}(n, m+j)\right\} x^{j} \\
& =\sum_{j=0}^{n}\left\{\sum_{m=j}^{n} \frac{(-1)^{m-j}\left(\begin{array}{c}
m \\
j
\end{array}\right)}{(m-j+1)^{k}} S_{1}(n, m)\right\} x^{j}
\end{aligned}
$$

which is the same as the expression in (24). From (1), we note that

$$
\frac{1}{\operatorname{Lif}_{k}(-t)} \widehat{c}_{n}^{(k)}(x) \sim\left(1, e^{t}-1\right), \quad x^{n} \sim(1, t) .
$$

For $n \geq 1$, by (19) and (28), we get

$$
\begin{aligned}
\frac{1}{\operatorname{Lif}_{k}(-t)} \widehat{c}_{n}^{(k)}(x) & =x\left(\frac{t}{e^{t}-1}\right)^{n} x^{-1} x^{n}=x\left(\frac{t}{e^{t}-1}\right)^{n} x^{n-1} \\
& =x B_{n-1}^{(n)}(x)=\sum_{l=0}^{n-1}\left(\begin{array}{c}
n-1 \\
l
\end{array}\right) B_{n-1-l}^{(n)} x^{l+1}
\end{aligned}
$$


Thus, by (29), we see that

$$
\begin{aligned}
\widehat{c}_{n}^{(k)}(x)= & \sum_{l=0}^{n-1}\left(\begin{array}{c}
n-1 \\
l
\end{array}\right) B_{n-1-l}^{(n)} \operatorname{Lif}_{k}(-t) x^{l+1} \\
= & \sum_{l=0}^{n-1} \sum_{m=0}^{l+1}(-1)^{m}\left(\begin{array}{c}
n-1 \\
l
\end{array}\right)\left(\begin{array}{c}
l+1 \\
m
\end{array}\right) \frac{B_{n-1-l}^{(n)}}{(m+1)^{k}} x^{l+1-m} \\
= & \sum_{l=0}^{n-1} \sum_{j=0}^{l+1}(-1)^{l+1-j}\left(\begin{array}{c}
n-1 \\
l
\end{array}\right)\left(\begin{array}{c}
l+1 \\
j
\end{array}\right) \frac{B_{n-1-l}^{(n)} x^{j}}{(l+2-j)^{k}} \\
= & \sum_{l=0}^{n-1}(-1)^{l+1}\left(\begin{array}{c}
n-1 \\
l
\end{array}\right) \frac{B_{n-1-l}^{(n)}}{(l+2)^{k}} \\
& +\sum_{j=1}^{n}\left\{\sum_{l=j-1}^{n-1}(-1)^{l+1-j}\left(\begin{array}{c}
n-1 \\
l
\end{array}\right)\left(\begin{array}{c}
l+1 \\
j
\end{array}\right) \frac{B_{n-1-l}^{(n)}}{(l+2-j)^{k}}\right\} x^{j} .
\end{aligned}
$$

Therefore, by (27) and (30), we obtain the following theorem.

Theorem 1 For $n \geq 1,1 \leq j \leq n$, we have

$$
\sum_{m=j}^{n} \frac{(-1)^{m-j}\left(\begin{array}{c}
m \\
j
\end{array}\right)}{(m-j+1)^{k}} S_{1}(n, m)=\sum_{l=j-1}^{n-1}(-1)^{l+1-j}\left(\begin{array}{c}
n-1 \\
l
\end{array}\right)\left(\begin{array}{c}
l+1 \\
j
\end{array}\right) \frac{B_{n-1-l}^{(n)}}{(l+2-j)^{k}}
$$

In addition, for $n \geq 1$, we have

$$
\widehat{c}_{n}^{(k)}=\sum_{m=0}^{n} S_{1}(n, m) \frac{(-1)^{m}}{(m+1)^{k}}=\sum_{l=0}^{n-1}(-1)^{l+1}\left(\begin{array}{c}
n-1 \\
l
\end{array}\right) \frac{B_{n-1-l}^{(n)}}{(l+2)^{k}} .
$$

From (18), we note that

$$
\widehat{c}_{n}^{(k)}(x+y)=\sum_{j=0}^{n}\left(\begin{array}{l}
n \\
j
\end{array}\right) \widehat{c}_{j}^{(k)}(x) p_{n-j}(y)
$$

where $p_{n}(y)=\frac{1}{\operatorname{Lif}_{k}(-t)} \widehat{c}_{n}^{(k)}(y) \sim\left(1, e^{t}-1\right)$.

By (22) and (23), we get

$$
(y)_{n}=p_{n}(y) \sim\left(1, e^{t}-1\right) .
$$

Thus, from (31) and (32), we have

$$
\widehat{c}_{n}^{(k)}(x+y)=\sum_{j=0}^{n}\left(\begin{array}{l}
n \\
j
\end{array}\right) \widehat{c}_{j}^{(k)}(x)(y)_{n-j}
$$

By (14), (16), and (21), we get

$$
\widehat{c}_{n}^{(k)}(x+1)-\widehat{c}_{n}^{(k)}(x)=\left(e^{t}-1\right) \widehat{c}_{n}^{(k)}(x)=n \widehat{c}_{n-1}^{(k)}(x) .
$$


For $s_{n}(x) \sim(g(t), f(t))$, the recurrence formula for $s_{n}(x)$ is given by

$$
s_{n+1}(x)=\left(x-\frac{g^{\prime}(t)}{g(t)}\right) \frac{1}{f^{\prime}(t)} s_{n}(x) \quad(\text { see }[10])
$$

By (21) and (34), we get

$$
\begin{aligned}
\widehat{c}_{n+1}^{(k)}(x) & =\left(x-\frac{\operatorname{Lif}_{k}^{\prime}(-t)}{\operatorname{Lif}_{k}(-t)}\right) e^{-t} \widehat{c}_{n}^{(k)}(x) \\
& =x \widehat{c}_{n}^{(k)}(x-1)-e^{-t} \frac{\operatorname{Lif}_{k}^{\prime}(-t)}{\operatorname{Lif}_{k}(-t)} \widehat{c}_{n}^{(k)}(x) .
\end{aligned}
$$

We observe that

$$
\begin{aligned}
\frac{\operatorname{Lif}_{k}^{\prime}(-t) \operatorname{Lif}_{k}^{\prime}(-t)}{\operatorname{Lif}_{k}(-t)} \widehat{c}_{n}^{(k)}(x) & =\operatorname{Lif}_{k}^{\prime}(-t) \frac{1}{\operatorname{Lif}_{k}(-t)} \widehat{c}_{n}^{(k)}(x)=\operatorname{Lif}_{k}^{\prime}(-t)(x)_{n} \\
& =\sum_{l=0}^{n} S_{1}(n, l) \operatorname{Lif}_{k}^{\prime}(-t) x^{l} \\
& =\sum_{l=0}^{n} S_{1}(n, l) \sum_{m=0}^{l} \frac{(-1)^{m}\left(\begin{array}{l}
l \\
m
\end{array}\right)}{(m+2)^{k}} x^{l-m} \\
& =\sum_{j=0}^{n}\left\{\sum_{l=j}^{n} \frac{(-1)^{l-j}\left(\begin{array}{l}
l \\
j
\end{array}\right)}{(l-j+2)^{k}} S_{1}(n, l)\right\} x^{j} .
\end{aligned}
$$

Therefore, by (35) and (36), we obtain the following theorem.

Theorem 2 For $n \geq 0$, we have

$$
\widehat{c}_{n+1}^{(k)}(x)=x \widehat{c}_{n}^{(k)}(x-1)-\sum_{j=0}^{n}\left\{\sum_{l=j}^{n} S_{1}(n, l) \frac{(-1)^{l-j}}{(l-j+2)^{k}}\left(\begin{array}{l}
l \\
j
\end{array}\right)\right\}(x-1)^{j}
$$

From (11), we note that

$$
\begin{aligned}
\widehat{c}_{n}^{(k)}(y)= & \left\langle\sum_{l=0}^{\infty} \widehat{c}_{l}^{(k)}(y) \frac{t^{l}}{l !} \mid x^{n}\right\rangle=\left\langle\operatorname{Lif}_{k}(-\log (1+t))(1+t)^{y} \mid x^{n}\right\rangle \\
= & \left\langle\operatorname{Lif}_{k}(-\log (1+t))(1+t)^{y} \mid x x^{n-1}\right\rangle \\
= & \left\langle\partial_{t}\left(\operatorname{Lif}_{k}(-\log (1+t))(1+t)^{y}\right) \mid x^{n-1}\right\rangle \\
= & \left\langle\partial_{t}\left(\operatorname{Lif}_{k}(-\log (1+t))\right)(1+t)^{y} \mid x^{n-1}\right\rangle \\
& +\left\langle\operatorname{Lif}_{k}(-\log (1+t)) \partial_{t}(1+t)^{y} \mid x^{n-1}\right\rangle \\
= & \left\langle\partial_{t}\left(\operatorname{Lif}_{k}(-\log (1+t))\right)(1+t)^{y} \mid x^{n-1}\right\rangle+\widehat{c}_{n-1}^{(k)}(y-1)
\end{aligned}
$$

where $\partial_{t} f(t)=\frac{d f(t)}{d t}$.

Since $t \operatorname{Lif}_{k}^{\prime}(t)=\operatorname{Lif}_{k-1}(t)-\operatorname{Lif}_{k}(t)$, we get

$$
\operatorname{Lif}_{k}^{\prime}(t)=\frac{\operatorname{Lif}_{k-1}(t)-\operatorname{Lif}_{k}(t)}{t}
$$


By (37) and (38), we see that

$$
\begin{aligned}
\widehat{c}_{n}^{(k)}(y)= & y \widehat{c}_{n-1}^{(k)}(y-1) \\
& +\left\langle\frac{\operatorname{Lif} f_{k-1}(-\log (1+t))-\operatorname{Lif}_{k}(-\log (1+t))}{(1+t) \log (1+t)}(1+t)^{y} \mid x^{n-1}\right\rangle \\
= & y \widehat{c}_{n-1}^{(k)}(y-1) \\
& +\left\langle\frac{\operatorname{Lif} f_{k-1}(-\log (1+t))-\operatorname{Lif}_{k}(-\log (1+t))}{t(1+t)}(1+t)^{y} \mid \frac{t}{\log (1+t)} x^{n-1}\right\rangle .
\end{aligned}
$$

From (1), (6), and (38), we note that

$$
\begin{aligned}
\widehat{c}_{n}^{(k)}(y)= & \widehat{y c}_{n-1}^{(k)}(y-1)+\sum_{l=0}^{n-1} \frac{B_{l}^{(l)}(1)}{l !}(n-1)_{l} \\
& \times\left\langle\frac{\operatorname{Lif}_{k-1}(-\log (1+t))-\operatorname{Lif}_{k}(-\log (1+t))}{t}(1+t)^{y-1} \mid x^{n-l-1}\right\rangle \\
= & \widehat{y}_{n-1}^{(k)}(y-1)+\sum_{l=0}^{n-1} \frac{B_{l}^{(l)}(1)}{l !}(n-1)_{l} \\
& \times\left\langle\frac{\operatorname{Lif}_{k-1}(-\log (1+t))-\operatorname{Lif}_{k}(-\log (1+t))}{t}(1+t)^{y-1} \mid t \frac{x^{n-l}}{n-l}\right\rangle \\
= & \widehat{\mathcal{c}}_{n-1}^{(k)}(y-1)+\sum_{l=0}^{n-1}\left(\begin{array}{c}
n-1 \\
l
\end{array}\right) \frac{B_{l}^{(l)}(1)}{n-l}\left\{\widehat{c}_{n-l}^{(k-1)}(y-1)-\widehat{c}_{n-l}^{(k)}(y-1)\right\} \\
= & y \widehat{c}_{n-1}^{(k)}(y-1)+\frac{1}{n} \sum_{l=0}^{n-1}\left(\begin{array}{c}
n \\
l
\end{array}\right) B_{l}^{(l)}(1)\left\{\widehat{c}_{n-l}^{(k-1)}(y-1)-\widehat{c}_{n-l}^{(k)}(y-1)\right\} .
\end{aligned}
$$

It is not difficult to show that $\widehat{c}_{0}^{(k)}(y-1)=\widehat{c}_{0}^{(k-1)}(y-1)$. Since $\hat{c}_{0}^{(k)}(y-1)=\widehat{c}_{0}^{(k-1)}(y-1)$, by (40), we obtain the following theorem.

Theorem 3 For $n \geq 1$, we have

$$
\widehat{c}_{n}^{(k)}(x)=x \hat{c}_{n-1}^{(k)}(x-1)+\frac{1}{n} \sum_{l=0}^{n}\left(\begin{array}{l}
n \\
l
\end{array}\right) B_{l}^{(l)}(1)\left\{\widehat{c}_{n-l}^{(k-1)}(x-1)-\widehat{c}_{n-l}^{(k)}(x-1)\right\} .
$$

For $n \geq m \geq 1$, we compute

$$
\left\langle(\log (1+t))^{m} \operatorname{Lif}_{k}(-\log (1+t)) \mid x^{n}\right\rangle
$$

in two different ways.

On the one hand,

$$
\begin{aligned}
& \left\langle(\log (1+t))^{m} \operatorname{Lif}_{k}(-\log (1+t)) \mid x^{n}\right\rangle \\
& \quad=\left\langle\operatorname{Lif}_{k}(-\log (1+t)) \mid \sum_{l=0}^{\infty} \frac{m !}{(l+m) !} S_{1}(l+m, m) t^{l+m} x^{n}\right\rangle
\end{aligned}
$$


On the other hand, we get

$$
\begin{aligned}
& \left\langle(\log (1+t))^{m} \operatorname{Lif}_{k}(-\log (1+t)) \mid x^{n}\right\rangle \\
& \quad=\left\langle(\log (1+t))^{m} \operatorname{Lif}_{k}(-\log (1+t)) \mid x x^{n-1}\right\rangle \\
& \quad=\left\langle\partial_{t}\left((\log (1+t))^{m} \operatorname{Lif}_{k}(-\log (1+t))\right) \mid x^{n-1}\right\rangle .
\end{aligned}
$$

Now, we observe that

$$
\begin{aligned}
\partial_{t}\left((\log (1+t))^{m} \operatorname{Lif}_{k}(-\log (1+t))\right) \\
=m(\log (1+t))^{m-1} \frac{1}{1+t} \operatorname{Lif}_{k}(-\log (1+t)) \\
\quad+(\log (1+t))^{m} \frac{\operatorname{Lif}_{k-1}(-\log (1+t))-\operatorname{Lif}_{k}(-\log (1+t))}{(1+t) \log (1+t)} \\
=(\log (1+t))^{m-1}(1+t)^{-1}\left\{m \operatorname{Lif}_{k}(-\log (1+t))\right. \\
\left.\quad+\operatorname{Lif}_{k-1}(-\log (1+t))-\operatorname{Lif}_{k}(-\log (1+t))\right\} .
\end{aligned}
$$

By (42) and (43), we get

$$
\begin{aligned}
\left\langle(\log (1+t))^{m} \operatorname{Lif}_{k}(-\log (1+t)) \mid x^{n}\right\rangle \\
=\sum_{l=0}^{n-m} \frac{(m-1) !}{(l+m-1) !} S_{1}(l+m-1, m-1) \\
\quad \times\left\{(m-1)\left\langle\operatorname{Lif}_{k}(-\log (1+t))(1+t)^{-1} \mid t^{l+m-1} x^{n-1}\right\rangle\right. \\
\left.\quad+\left\langle\operatorname{Lif}_{k-1}(-\log (1+t))(1+t)^{-1} \mid t^{l+m-1} x^{n-1}\right\rangle\right\} \\
=(m-1) \sum_{l=0}^{n-m} \frac{(m-1) !}{(l+m-1) !} S_{1}(l+m-1, m-1)(n-1)_{l+m-1} \\
\quad \times\left\langle\operatorname{Lif}_{k}(-\log (1+t))(1+t)^{-1} \mid x^{n-m-l}\right\rangle \\
\quad+\sum_{l=0}^{n-m} \frac{(m-1) !}{(l+m-1) !} S_{1}(l+m-1, m-1)(n-1)_{l+m-1} \\
\quad \times\left\langle\operatorname{Lif}_{k-1}(-\log (1+t))(1+t)^{-1} \mid x^{n-m-l}\right\rangle \\
=\sum_{l=0}^{n-m}(m-1) !\left(\begin{array}{c}
n-1 \\
l+m-1
\end{array}\right) S_{1}(l+m-1, m-1) \\
\quad \times\left\{(m-1) \widehat{c}_{n-l-m}^{(k)}(-1)+\widehat{c}_{n-l-m}^{(k-1)}(-1)\right\} .
\end{aligned}
$$

Therefore, by (41) and (44), we obtain the following theorem. 
Theorem 4 For $n \geq m \geq 1$, we have

$$
\begin{aligned}
\sum_{l=0}^{n-m} m !\left(\begin{array}{c}
n \\
l+m
\end{array}\right) S_{1}(l+m, m) \widehat{c}_{n-l-m}^{(k)} \\
=\sum_{l=0}^{n-m}(m-1) !\left(\begin{array}{c}
n-1 \\
l+m-1
\end{array}\right) S_{1}(l+m-1, m-1) \\
\quad \times\left\{(m-1) \widehat{c}_{n-l-m}^{(k)}(-1)+\widehat{c}_{n-l-m}^{(k-1)}(-1)\right\} .
\end{aligned}
$$

In particular, if we take $m=1$, then we get

$$
\widehat{c}_{n}^{(k-1)}(-1)=\sum_{l=0}^{n-1}(-1)^{l} l !\left(\begin{array}{c}
n \\
l+1
\end{array}\right) \widehat{c}_{n-l-1}^{(k)}
$$

Remark For $s_{n}(x) \sim(g(t), f(t))$, it is known that

$$
\frac{d}{d x} s_{n}(x)=\sum_{l=0}^{n-1}\left(\begin{array}{l}
n \\
l
\end{array}\right)\left\langle\bar{f}(t) \mid x^{n-l}\right\rangle s_{l}(x) \quad(\text { see }[10, \mathrm{p} .108])
$$

By (21) and (45), we easily show that

$$
\frac{d}{d x} \widehat{c}_{n}^{(k)}(x)=(-1)^{n} n ! \sum_{l=0}^{n-1} \frac{(-1)^{l-1}}{(n-l) l !} \widehat{c}_{l}^{(k)}(x),
$$

which is a special case of Proposition 2 in [4].

Let us consider the following two Sheffer sequences:

$$
\widehat{c}_{n}^{(k)}(x) \sim\left(\frac{1}{\operatorname{Lif}_{k}(-t)}, e^{t}-1\right),
$$

and

$$
B_{n}^{(r)}(x) \sim\left(\left(\frac{e^{t}-1}{t}\right)^{r}, t\right) .
$$

Suppose that

$$
\widehat{c}_{n}^{(k)}(x)=\sum_{m=0}^{n} C_{n, m} B_{m}^{(r)}(x) .
$$

By (20), we see that

$$
\begin{aligned}
C_{n, m} & =\frac{1}{m !}\left\langle\frac{\left(\frac{t}{\log (1+t)}\right)^{r}}{\frac{1}{\operatorname{Lif}_{k}(-\log (1+t))}}(\log (1+t))^{m} \mid x^{n}\right\rangle \\
& =\frac{1}{m !}\left\langle\operatorname{Lif}_{k}(-\log (1+t))\left(\frac{t}{\log (1+t)}\right)^{r}(\log (1+t))^{m} \mid x^{n}\right\rangle
\end{aligned}
$$




$$
\begin{aligned}
= & \frac{1}{m !} \sum_{l=0}^{n-m} \frac{m !}{(l+m) !} S_{1}(l+m, m)(n)_{l+m} \\
& \times\left\langle\operatorname{Lif}_{k}(-\log (1+t))\left(\frac{t}{\log (1+t)}\right)^{r} \mid x^{n-l-m}\right\rangle \\
= & \frac{1}{m !} \sum_{l=0}^{n-m} \frac{m !}{(l+m) !} S_{1}(l+m, m)(n)_{l+m} \sum_{a=0}^{n-l-m} B_{a}^{(a-r+1)} \frac{1}{a !} \\
& \times\left\langle\operatorname{Lif}_{k}(-\log (1+t)) \mid t^{a} x^{n-l-m}\right\rangle \\
= & \sum_{l=0}^{n-m}\left(\begin{array}{c}
n \\
l+m
\end{array}\right) S_{1}(l+m, m) \sum_{a=0}^{n-l-m} B_{a}^{(a-r+1)} \frac{(n-l-m)_{a}}{a !} \\
& \times\left\langle\operatorname{Lif}_{k}(-\log (1+t)) \mid x^{n-l-m-a}\right\rangle \\
& \sum_{l=0}^{n-m} \sum_{a=0}^{n-l-m}\left(\begin{array}{c}
n \\
l+m
\end{array}\right)\left(\begin{array}{c}
n-m-l \\
a
\end{array}\right) S_{1}(l+m, m) B_{a}^{(a-r+1)}(1) c_{n-l-m-a}^{(k)} .
\end{aligned}
$$

Therefore, by (47) and (48), we obtain the following theorem.

Theorem 5 For $n \geq 0$, we have

$$
\widehat{c}_{n}^{(k)}(x)=\sum_{m=0}^{n}\left\{\sum_{l=0}^{n-m} \sum_{a=0}^{n-m-l}\left(\begin{array}{c}
n \\
l+m
\end{array}\right)\left(\begin{array}{c}
n-m-l \\
a
\end{array}\right) S_{1}(l+m, m) B_{a}^{(a-r+1)}(1) \widehat{c}_{n-m-l-a}^{(k)}\right\} B_{m}^{(r)}(x) .
$$

Remark The Narumi polynomials of order $a$ are defined by the generating function to be

$$
\sum_{k=0}^{\infty} \frac{N_{k}^{(a)}(x)}{k !} t^{k}=\left(\frac{t}{\log (1+t)}\right)^{-a}(1+t)^{x} \quad(\text { see }[10, \mathrm{p} .127])
$$

Indeed, $N_{a}^{(k)}(x)=B_{k}^{(k+a+1)}(x+1), N_{k}^{(a)}(x) \sim\left(\left(\frac{e^{t}-1}{t}\right)^{a}, e^{t}-1\right)$.

By (48) and (49), we get

$$
C_{n, m}=\sum_{l=0}^{n-m} \sum_{a=0}^{n-m-l}\left(\begin{array}{c}
n \\
l+m
\end{array}\right)\left(\begin{array}{c}
n-l-m \\
a
\end{array}\right) S_{1}(l+m, m) N_{a}^{(-r)} \widehat{c}_{n-l-m-a}^{(k)} .
$$

From (47) and (50), we have

$$
\begin{aligned}
& \widehat{c}_{n}^{(k)}(x)=\sum_{m=0}^{n}\left\{\sum_{l=0}^{n-m} \sum_{a=0}^{n-m-l}\left(\begin{array}{c}
n \\
l+m
\end{array}\right)\left(\begin{array}{c}
n-l-m \\
a
\end{array}\right)\right. \\
& \left.\times S_{1}(l+m, m) N_{a}^{(-r)} \widehat{c}_{n-l-m-a}^{(k)}\right\} B_{m}^{(r)}(x) .
\end{aligned}
$$


By (1), we easily show that

$$
\begin{aligned}
C_{n, m}= & \sum_{l=0}^{n-m} \sum_{a=0}^{n-m-l} \sum_{a_{1}+\cdots+a_{r}=a}\left(\begin{array}{c}
n \\
l+m
\end{array}\right)\left(\begin{array}{c}
n-l-m \\
a
\end{array}\right)\left(\begin{array}{c}
a \\
a_{1}, \ldots, a_{r}
\end{array}\right) \\
& \times S_{1}(l+m, m) b_{a_{1}} \cdots b_{a_{r}} \widehat{c}_{n-m-l-a}^{(k)} .
\end{aligned}
$$

From (47) and (52), we can derive the following equation:

$$
\begin{aligned}
& \widehat{c}_{n}^{(k)}(x)=\sum_{m=0}^{n}\left\{\sum_{l=0}^{n-m} \sum_{a=0}^{n-m-l} \sum_{a_{1}+\cdots+a_{r}=a}\left(\begin{array}{c}
n \\
l+m
\end{array}\right)\left(\begin{array}{c}
n-l-m \\
a
\end{array}\right)\left(\begin{array}{c}
a \\
a_{1}, \ldots, a_{r}
\end{array}\right)\right. \\
& \left.\times S_{1}(l+m, m)\left(\prod_{i=1}^{r} b_{a_{i}}\right) \widehat{c}_{n-m-l-a}^{(k)}\right\} B_{m}^{(r)}(x) .
\end{aligned}
$$

For (20) and (24), let

$$
\widehat{c}_{n}^{(k)}(x)=\sum_{m=0}^{n} C_{n, m} H_{m}^{(r)}(x \mid \lambda)
$$

where, by (20), we get

$$
\begin{aligned}
C_{n, m}= & \frac{1}{m !(1-\lambda)^{r}}\left\langle\operatorname{Lif}_{k}(-\log (1+t))(1+t-\lambda)^{r} \mid(\log (1+t))^{m} x^{n}\right\rangle \\
= & \frac{1}{m !(1-\lambda)^{r}} \sum_{l=0}^{n-m} \frac{m !}{(l+m) !} S_{1}(l+m, m)(n)_{l+m} \\
& \times\left\langle\operatorname{Lif}_{k}(-\log (1+t))(1+t-\lambda)^{r} \mid x^{n-l-m}\right\rangle .
\end{aligned}
$$

We observe that

$$
\begin{aligned}
& \left\langle\operatorname{Lif}_{k}(-\log (1+t))(1+t-\lambda)^{r} \mid x^{n-l-m}\right\rangle \\
& =\sum_{a=0}^{r}\left(\begin{array}{l}
r \\
a
\end{array}\right)(1-\lambda)^{r-a}\left\langle\operatorname{Lif}_{k}(-\log (1+t)) \mid t^{a} x^{n-l-m}\right\rangle \\
& =\sum_{a=0}^{r}\left(\begin{array}{l}
r \\
a
\end{array}\right)(1-\lambda)^{r-a}(n-m-l)_{a}\left\langle\operatorname{Lif}_{k}(-\log (1+t)) \mid x^{n-l-m-a}\right\rangle \\
& =\sum_{a=0}^{r}\left(\begin{array}{l}
r \\
a
\end{array}\right)(1-\lambda)^{r-a}(n-m-l)_{a} \widehat{c}_{n-l-m-a}^{(k)} .
\end{aligned}
$$

Thus, by (55) and (56), we get

$$
C_{n, m}=\sum_{l=0}^{n-m} \sum_{a=0}^{r}\left(\begin{array}{c}
n \\
l+m
\end{array}\right)\left(\begin{array}{l}
r \\
a
\end{array}\right)(n-m-l)_{a}(1-\lambda)^{-a} S_{1}(l+m, m) \widehat{c}_{n-m-l-a}^{(k)} .
$$

Therefore, by (54) and (57), we obtain the following theorem. 
Theorem 6 For $n \geq 0$, we have

$$
\begin{aligned}
& \widehat{c}_{n}^{(k)}(x)=\sum_{m=0}^{n}\left\{\sum_{l=0}^{n-m} \sum_{a=0}^{r}\left(\begin{array}{c}
n \\
l+m
\end{array}\right)\left(\begin{array}{l}
r \\
a
\end{array}\right)(n-m-l)_{a}(1-\lambda)^{-a} S_{1}(l+m, m)\right. \\
& \left.\times \widehat{c}_{n-m-l-a}^{(k)}\right\} H_{m}^{(r)}(x \mid \lambda) \text {. }
\end{aligned}
$$

For $\widehat{c}_{n}^{(k)}(x) \sim\left(\frac{1}{\operatorname{Lif}_{k}(-t)}, e^{t}-1\right)$, and $(x)_{n} \sim\left(1, e^{t}-1\right)$, let us assume that

$$
\widehat{c}_{n}^{(k)}(x)=\sum_{m=0}^{n} C_{n, m}(x)_{m} .
$$

From (20), we note that

$$
\begin{aligned}
C_{n, m} & =\frac{1}{m !}\left\langle\operatorname{Lif}_{k}(-\log (1+t)) t^{m} \mid x^{n}\right\rangle \\
& =\frac{1}{m !}\left\langle\operatorname{Lif}_{k}(-\log (1+t)) \mid t^{m} x^{n}\right\rangle \\
& =\left(\begin{array}{c}
n \\
m
\end{array}\right)\left\langle\operatorname{Lif}_{k}(-\log (1+t)) \mid x^{n-m}\right\rangle \\
& =\left(\begin{array}{c}
n \\
m
\end{array}\right) \widehat{c}_{n-m}^{(k)} .
\end{aligned}
$$

Therefore, by (58) and (59), we obtain the following theorem.

Theorem 7 For $n \geq 0$, we have

$$
\widehat{c}_{n}^{(k)}(x)=\sum_{m=0}^{n}\left(\begin{array}{l}
n \\
m
\end{array}\right) \widehat{c}_{n-m}^{(k)}(x)_{m} .
$$

\section{Competing interests}

The authors declare that they have no competing interests.

\section{Authors' contributions}

All authors contributed equally to the manuscript and typed, read, and approved the final manuscript.

\section{Author details}

${ }^{1}$ Department of Mathematics, Sogang University, Seoul, 121-742, Republic of Korea. ${ }^{2}$ Department of Mathematics, Kwangwoon University, Seoul, 139-701, Republic of Korea.

\section{Acknowledgements}

The authors express their sincere gratitude to the referees for their valuable suggestions and comments. This work was supported by the National Research Foundation of Korea (NRF) grant funded by the Korea government (MOE) (No. 2012R1A1A2003786)

\section{Received: 8 August 2013 Accepted: 7 January 2014 Published: 27 Jan 2014}

\section{References}

1. Jordan, C: Sur des polynomes analogues aux polynomes de Bernoulli et sur des formules de sommation analogues à celle de MacLaurin-Euler. Acta Sci. Math. 4, 130-150 (1928/1929)

2. Komatsu, T: Poly-Cauchy numbers. RIMS Kokyuroku 1806, $42-53$ (2012). Available at http://www.kurims.kyoto-u.ac.jp/ kyodo/kokyuroku/contents/pdf/1806-06.pdf

3. Komatsu, T: Poly-Cauchy numbers. Kyushu J. Math. 67, 143-153 (2013) 
4. Komatsu, T: Poly-Cauchy numbers with a q parameter. Ramanujan J. 31, 353-371 (2013)

5. Komatsu, T: Sums of products of Cauchy numbers including poly-Cauchy numbers. J. Discrete Math. 2013, Article ID 373927 (2013)

6. Komatsu, T, Liptai, K, Szalay, L: Some relationships between poly-Cauchy type numbers and poly-Bernoulli type numbers. East-West J. Math. 14(2), 114-120 (2012)

7. Komatsu, T, Luca, F: Some relationships between poly-Cauchy numbers and poly-Bernoulli numbers. Ann. Math. Inform. 41, 99-105 (2013)

8. Nörlund, NE: Vorlesungen über Differenzenrechnung. Springer, Berlin (1924)

9. Erdélyi, A, Magnus, W, Overhettinger, F, Tricomi, FG: Higher Transcendental Functions, vol. 3. McGraw-Hill, New York (1955)

10. Roman, S: The Umbral Calculus. Dover, New York (2005). ISBN:0-486-44139-3

11. Araci, S, Acikgoz, M: A note on the Frobenius-Euler numbers and polynomials associated with Bernstein polynomials. Adv. Stud. Contemp. Math. 22(3), 399-406 (2012)

12. Kim, DS, Kim, T, Lee, SH: Poly-Cauchy numbers and polynomials with umbral calculus wiewpoint. Int. J. Math. Anal. 7, 2235-2253 (2013)

13. Kim, T: Some identities on the $q$-Euler polynomials of higher order and $q$-Stirling numbers by the fermionic $p$-adic integral on $\mathbb{Z}_{p}$. Russ. J. Math. Phys. 16(4), 484-491 (2009)

14. Carliz, L: A note on Bernoulli and Euler polynomials of the second kind. Scr. Math. 23, 323-330 (1961)

15. Roman, SM, Rota, G-C: The umbral calculus. Adv. Math. 27(2), 95-188 (1978)

10.1186/1687-1847-2014-36

Cite this article as: Kim and Kim: Poly-Cauchy numbers and polynomials of the second kind with umbral calculus viewpoint. Advances in Difference Equations 2014, 2014:36

\section{Submit your manuscript to a SpringerOpen ${ }^{\ominus}$ journal and benefit from:}

- Convenient online submission

- Rigorous peer review

- Immediate publication on acceptance

- Open access: articles freely available online

- High visibility within the field

- Retaining the copyright to your article 